\title{
Comprehensive analysis of micro RNAs in recurrent implantation failures patients and construction of prediction models based on circulating micro RNAs
}

\section{Peigen Chen}

Sixth Affiliated Hospital of Sun Yat-sen University

\section{Yingchun Guo}

Sixth Affiliated Hospital of Sun Yat-sen University

Tingting Li

Sixth Affiliated Hospital of Sun Yat-sen University

Lei Jia

Sixth Affiliated Hospital of Sun Yat-sen University

\section{Yanfang Wang}

Sixth Affiliated Hospital of Sun Yat-sen University

Yi Zhou

Sixth Affiliated Hospital of Sun Yat-sen University

Cong Fang ( $\nabla$ fangcong@mail.sysu.edu.cn )

Sixth Affiliated Hospital of Sun Yat-sen University

\section{Research Article}

Keywords: Recurrent implantation failure, circulating micro RNA, prediction, competing endogenous RNAs, assisted reproductive technology, GEO

Posted Date: January 15th, 2021

DOI: https://doi.org/10.21203/rs.3.rs-145125/v1

License: (c) (1) This work is licensed under a Creative Commons Attribution 4.0 International License. Read Full License 


\section{Abstract}

\section{Background}

Recurrent implantation failure (RIF) is an obstacle in the process of assisted reproductive technology (ART). At present, there was limited research on its pathogenesis, diagnosis and treatment methods.

\section{Results}

In this study, a series of analytical tools were used to analyze differences in miRNAs, mRNAs and IncRNAs in the endometrium of patients in the RIF group and the control group. At the same time, multiple databases are used to predict the target genes of non-coding RNAs. Then the competing endogenous RNA (ceRNA) network was built to describe the relationship between gene regulation in the endometrium of the RIF.

\section{Concludes}

Based on the results of the logistic regression of co-expression miRNAs between serum and endometrial samples, we built a predictive model based on circulating miRNAs.

\section{Introduction}

Recurrent implantation failure (RIF) is a thorny issue that couples undergoing in vitro fertilization (IVF) /intracytoplasmic sperm injection (ICSI) may face. The generally accepted definition is that women under the age of 40 have transferred at least four high-quality embryos in at least three fresh or frozen cycles or have transferred a total of 10 high-quality embryos but have not yet achieved clinical pregnancy $(1-3)$. Though along with improving in vitro fertilization embryo transfer (IVF-ET) technology and increasing clinical pregnancy rate, recurrent implantation failure is still a tough problem in the process of IVF-ET. The normal embryo implantation generally only occurs during the window of implantation (WOI)(4) which refers to days 20-24 of the normal menstrual cycle. Abnormalities of the endometrium at this stage were important factors that lead to RIF.

MicroRNAs (miRNA) are a class of non-coding RNA molecules with a length of about 22 nucleotides that are widely found in eukaryotic cells. It plays an important role in the post-transcriptional regulation of genes, which includes targeting mRNA, negatively by mRNA cleavage(5), releasing translational suppression(6), and targeting gene promoters(7). There had been some studies confirming the role of miRNA in endometrial regulation (8-11). For example, miR-30b, miR-30d, and miR-494 had been reported to play an important role in the regulation of endometrial function (8). Recent researches reported miRNAs associated with RIF, such as miR-34c-5p(12) and miR-148A-3P(13). But the related mechanism was still unclear, and further in-depth studies were still lacking.

MicroRNAs not only exist in human cells, but also widely exist in extracellular body fluids, such as plasma, serum, urine and saliva(14). Peripheral blood miRNA is stable and can resist freezing and 
thawing at $4{ }^{\circ} \mathrm{C}$ and $-80^{\circ} \mathrm{C}(15)$. The stability is due to the binding with protein complexes, mainly Argonaute2 protein complexes, resulting in a protective effect on miRNAs (16). This characteristic makes peripheral blood miRNA a more stable biomarker for disease diagnosis(17). Fatemeh Azhari et. al showed that hsa-miR-145 and hsa-miR-23b were significantly down-regulated in both serum samples and endometrial samples of RIF patients (18). However, they did not describe the correlation in miRNA expression between serum and endometrium samples, nor the related regulatory mechanism.

Moreover, the regulatory effect of miRNA on mRNA can be regulated by LncRNA through competitive binding, which is called the endogenous RNA (ceRNA) network hypothesis(19). In the present study, we aim to use a larger sample size of data in our analysis to explore the regulatory molecular mechanism in endometrium of RIF patients at the WOI stage. At the same time, we aim to look for peripheral blood miRNAs closely related to RIF and provide a new way for non-invasive early diagnosis for RIF, thereby improving the clinical outcome of patients.

\section{Method}

We used R software (version 3.6.3) (Team, 2018), GraphPad Prism (version 8) and Bioconductor (Gentleman et al., 2004) for all statistical analyses in our study. The workflow was showed in Fig.1.

\section{Data acquisition and preprocessing}

The raw data of endometrial expression profile and corresponding clinical data of RIF group and control group (GSE71331, GSE71332) were obtained from the Gene Expression Omnibus (GEO) database (http://www.ncbi.nlm.nih.gov/geo). Paired serum and endometrial miRNA expression profile data were extracted from GSE108966.

To ensure consistency in the results of the analysis, we started with raw data of the dataset. Due to the different character of the platforms, the raw data of GSE71331 and GSE71332 were processed by "Limma" R package(20) (Agilent-052909 CBC IncRNA mRNA V3, Agilent-046064 Unrestricted Human miRNA V19.0), and the raw data of GSE108966 was processed by "Deseq2" R package(21) (Illumina HiSeq 2500).

\section{Selection of differentially expressed genes (DEGs)}

The scanning of differentially expressed genes (DE miRNA, DE IncRNA, DE miRNA) was performed by using the "limma" R package (GSE71331 and GSE71332) (20) and Deseq2 (21) (GSE108966) with the following criteria: $p$ value $<0.05$ and $\|$ log 2 -fold changel $>1$.

\section{Weighted correlation network analysis (WGCNA) of miRNA of endometria samples}

With the "WGCNA" R package (22), WGCNA was performed on differentially expressed miRNAs which selected based on GSE71332 dataset. The workflow of WGCNA was as follows. First, outliers were reduced by filtering the RNA-seq data. The coexpression matrix was constructed based on the absolute 
points of the relationships between gene expression levels. Then, we constructed a Pearson correlation matrix of paired genes, and a weighted adjacency matrix was developed based on the power function (where is the Pearson correlation between gene $b$ and gene $d$, is the adjacency between gene $b$ and gene $d$, and $\beta$ emphasizes the strength of the relationship between the genes). Then, we selected an optimal $\beta$ value to develop a scale-free coexpression network and a similarity matrix. Next, a topological overlap matrix (TOM) was constructed from the adjacency matrix. We performed average linkage hierarchical clustering with a minimum gene dendrogram size of 40 by using TOM-based dissimilarity measurements. By analyzing the modules, we calculated the dissimilarity and constructed module dendrograms for these modules.

We then calculated gene significance (GS) to estimate the significance of each module and measure the relationships between genes and sample traits. In the principal component analysis, module eigengenes (MEs) were considered as the main components, and we summarized gene expression levels as a single feature within a given module. Then, the $p$-value of the linear regression between gene expression levels and clinical feature were transformed, and the result was defined as the GS value $(\lg P=\mathrm{GS})$. Next, to estimate the relationship between the module and sample traits, we calculated the average value of GS, which was then defined as the module significance (MS). We used the relevant $p$-value to determine statistical significance. Next, clinical data including first trimester losses and live births were combined with gene modules for further analysis.

For the identification of key genes, the GS and module membership (MM, the correlation between the genes in the module and their expression profiles) of every key gene were calculated with the following thresholds: cor. gene GS > 0.5 and cor. gene $\mathrm{MM}>0.8$.

\section{Prediction of target IncRNAs/mRNAs of RIF related DE miRNAs}

The intersection of the differentially expressed genes and the genes of the key modules related to RIF in WGCNA was taken as RIF related DE miRNAs. Then miRDB(http://mirdb.org/miRDB/)(23), miRTarBase (http://mirtarbase.mbc.nctu.edu.

tw/) (24), and TargetScan (http://targetscan.org/)(25) were used to predict miRNA-targeting mRNAs. NPInter (http://bigdata.ibp.ac.cn/npinter4/)(26) and DIANA-LncBase(27) were used to predict miRNAtargeting IncRNAs. The intersection of differentially mRNAs/IncRNAs in GSE71331 and the miRNAtargeting mRNAs/IncRNAs were taken as targeting-DE mRNAs/IncRNAs.

\section{Construction of IncRNA-miRNA-mRNA regulatory network}

LncRNA-miRNA interactomes were then built based on targeting-DE IncRNAs and RIF related DE miRNAs. Similarly, mRNA-miRNA interactomes were built. Subsequently, IncRNA-miRNA-mRNA regulatory networks were constructed by using cytoscape, version 3.8 (https://cytoscape.org/). Key modules were selected by $\operatorname{MCODE}(28)$.

\section{Selection and validation of co-expression miRNAs between serum and endometrial samples}


The intersection of endometrium DE miRNAs and serum DE miRNAs was taken as intersection miRNAs. To ensure that their expressions were relevant, Pearson correlation analysis is performed by using GraphPad Prism (version 8). Genes with the Pearson correlation coefficient $|r| \geq 0.5$ were considered to be co-expressed miRNAs between serum and endometrium. And the intersection miRNAs were then validated in GSE71332.

\section{Prediction of target IncRNAs/mRNAs of co-expression miRNAs}

miRDB(http://mirdb.org/miRDB/)(23), miRTarBase (http://mirtarbase.mbc.

nctu.edu.tw/) (24), and TargetScan (http://targetscan.org/)(25) were used to predict miRNA-targeting mRNAs of co-expression miRNAs.

\section{Functional enrichment analysis of targeted DE mRNAs}

To understand the biological function of targeted DE mRNAs of GSE71332 and targeted mRNAs of GSE108966, Metascape (29) (http://metascape.org), which includes abundant functional annotations, such as KEGG pathway, Reactome pathway, canonical pathway, GO biological process, and CORUM (the comprehensive resource of mammalian protein complexes) annotations, was then used to perform functional enrichment analysis with a $p$-value of $<0.01$ as the cutoff value (29). Selected terms with a $p$ value of $<0.01$ and a number of genes greater than or equal to 3 were considered significant terms.

\section{Transcriptional Regulatory Relationship analysis of targeted DE mRNAs}

TRRUST (transcriptional regulatory relationships unravelled by sentence-based text-mining, http://www.grnpedia.org/trrust) is a TF-target regulatory interactions database based on the manual curation of Medline abstracts (30). TRRUST was subsequently used to screen transcription factors related to targeted DE mRNAs and targeted mRNAs and study their transcription regulation relationships.

\section{Causal relationship analysis}

DisNor (31) (https://disnor.uniroma2.it/) is a web-based tool that can generate and explore protein interaction networks based on disease genes using Mentha protein interaction data and causal interaction information annotated by SIGNOR. DisNor was used to explore the causal relationships among targeted DE mRNAs.

\section{Construction and validation of nomogram based on circulating miRNAs}

Logistic regression analysis was then performed with three selected factors by using "survival" $\mathrm{R}$ package(32) to select the best fit model. Then a nomogram was built to predict the risk of RIF patients by using "rms" R package. At the same time, the consistency index (C-index) was calculated to evaluate the model's ability to distinguish. The consistency of the predicted probability and the actual probability of the model was evaluated by the Calibration curves. The predictive performance of the model was 
evaluated by drawing the receiver operating characteristic (ROC) curve and calculating the area under the curve (AUC) values.

\section{Result}

\section{Clinical characteristics of samples used in the study}

All data came from samples taken during the window of implantation. The clinical characteristics of RIF group and control group in GSE71331 and GSE71332 were listed in Table 1. Totally, the mRNA and IncRNA expression profiles of 7 RIF samples and 5 control samples were extracted from GSE71331 and the corresponding miRNA expression profile were extracted from GSE71332. 56 patients (24 RIF patients and 32 control patients) with paired serum and endometrial miRNA expression profile data were extracted from GSE108966, including 24 RIF patients (age: 26-40 (34.96 \pm 3.93$), \mathrm{LH}+8$ ) and 32 control patients (age: 23-36 (29.63 \pm 3.65$)$, LH+8).

\section{Selection of differentially expressed genes (DEGs) in GSE71331/71332}

70 down-regulation IncRNAs and 67 up-regulation IncRNAs were selected by "limma" package with pvalue $<0.05$ and |log 2-fold change| $>1$. Similarly, 122 down-regualtion mRNAs, 184 up-regulation mRNAs, 6 down-regulation miRNAs and 103 up-regulation miRNAs were selected (supplement table 1).

\section{Selection of RIF related miRNAs by WGCNA in GSE71332}

A gene coexpression network was then constructed based on the samples of GSE71332 by WGCNA to select the most significant gene modules and genes. This procedure can also help to elucidate the relationship between genes and clinical features. We first eliminated the outlier samples, showing the highest variance in the module, for further analysis. Next, a scale-free network was constructed with a soft threshold of $\beta=7$, and 16 modules were selected with a minimum module size of 50 for further analysis (Fig. 2 B).

Then, the overall expression gene level was taken as the MS to estimate the relationship between the corresponding modules and clinical phenotypes (Fig. 2 A). Based on the results, we found that the turquoise module showed the most significant positive correlation with the RIF (cor $=0.97, p<0.0001$ ) (Fig.2 C). Therefore, the turquoise module was chosen as RIF related modules.

Finally, 97 intersection miRNAs between DEG and WGCNA were selected as RIF-related DE miRNAs (supplement table 3).

\section{Construction of IncRNA-miRNA-mRNA regulatory network}

Based on the interaction of the prediction of 3 databases (miRDB, miTarBase, TargetScan) and DE mRNAs, 45 mRNAs were selected for network construction. Similarly, 7 IncRNAs were selected (supplement table 4). Finally, a IncRNA-miRNA-mRNA regulatory network was constructed based on 80 
miRNAs, 45 mRNAs and 7 IncRNAs by using cytoscape (Fig. 3 A, C). By using MCODE app of cytoscape, key module network was selected (Fig.3 B).

\section{Functional enrichment analysis and causal relationship analysis}

By using Metascape, we found that the targeted DE mRNAs were enriched mainly in terms of cell adhesion mediated by integrin (GO: 0033627), female pregnancy (GO: 0007565), fatty acid metabolic process (GO: 0006631) and reproductive structure development (GO: 0048608) (Fig.4 C). The pathways targeted DE mRNAs enriched in included focal adhesion, signaling by nuclear receptors, MAPK pathway and PIP3 activates AKT pathway (Fig.4 D).

Key modules were selected in functional network (Fig.4A) by using MCODE cytoscape app. We found that one of the key modules was closely related with reproduction function (Fig.4B red circle), and their fist neighbor nodes were also drawn in Fig.4 B (outside the red circle).

According to TRRUST database, DYRK1A targeted ACADSB, ANK3, ASPH, HOXA13, ITGA3, and NPR3. SOX10 targeted ACADSB, ANK3, LAMB3, and SOX5 (Fig.4 E).

By using the DisNor tool, we screened the first neighboring genes of the targeted DE mRNAs, and built a causal relationship network (Fig.5). We found that these genes were closely related to MAPK signal pathway as we could see in the results of functional enrichment analysis.

\section{Selection of co-expression miRNAs between serum and endometrial samples and functional enrichment analysis}

For GSE108966, 63 down-regulation miRNAs and 45 up-regulation miRNAs were selected from endometrium samples by Deseq2 with the following criteria: $p$ value $<0.05$ and $\|$ log 2 -fold change| $>1$ (Fig. 6 A). Similarly, 28 down-regulation miRNAs and 22 up-regulation miRNAs were selected from serum samples (Fig. 6 B).

Hsa-miR-378e and hsa-miR-96-5p were selected as co-expression miRNAs between serum and endometrial samples (Fig.6 C).

Similarly, we used multiple databases to predict their target mRNAs (supplement table 5) and use the metascape tool for functional enrichment analysis. The results showed that the targeted mRNAs of hsamiR-96-5p mainly enriched in the terms of cellular response to organonitrogen compound, negative regulation of cell differentiation and regulation of protein serine/ threonine kinase activity (Fig.7 A). Fig.7 $B$ showed the pathway of the targeted mRNAs of hsa-miR-96-5p. We also noticed that the targeted mRNAs of hsa-miR-378e mainly enriched in protein serine/ threonine/ tyrosine kinase activity and regulation of organ growth (Fig.7 C). The pathway terms that the targeted mRNAs of hsa-miR-378e enriched in were cell cycle, Mitotic (Fig.7 D).

\section{Construction and validation of nomogram based on circulating miRNAs}


Based on the results of logistic regression analysis, the best fit models included age, the expression of hsa-miR-96-5p, and the expression of hsa-miR-378e. A nomogram with C-index: 0.865 was established to act as a prediction tool of RIF (Fig.8 A), which means that our model has a good ability to distinguish. The calibration curves in Fig. 8 B showed a good consistency of the predicted probability and the actual probability of the model with mean absolute error as 0.028 , mean squared error as 0.00096 and 0.9 quantile of absolute error as 0.044 . Fig. $8 \mathrm{C}$ showed the receiver operating characteristic (ROC) curve of the model, and the area under the curve (AUC) values was 0.865 , which means a promising predictive performance.

\section{Discussion}

Epigenetic regulation of gene expression played an important role in the development of recurrent implantation failure (RIF), and one of the most important parts was miRNA. As a previous study showed, the expression levels of miRNAs in endometrium and serum were difference during the menstrual cycle. Several factors, including different miRNAs, were selected as key molecules of regulation of endometrial acceptance and implantation (33). However, there was still a lack of research on the molecules that affect endometrial tolerance before implantation and the mechanisms of early dialogue between the embryo and the uterus (33). At present, more and more studies were beginning to pay attention to the diagnostic predictive value of circulating miRNA $(34,35)$. A research by Fatemeh Azhari et al. studied previously reported endometrial-specific miRNAs based on PCR technology in endometrial and serum samples from RIF patients. They found has-miR-145 and has-miR-23b were both down-regulated in endometrial and serum samples (18). Due to the limitations of the sample and detection technology, they could only study the known potentially meaningful miRNAs and could not explore them in depth.

In this study, by using WGCNA, we built a IncRNA-miRNA-mRNA regulatory network to analyze the expression and regulation characteristics of miRNAs in the endometrium and serum. By using MCODE app in cytoscape, two key modules were selected. We noticed that both hsa-miR-23a and hsa-miR-23b were interacted with ACADSB, DUSP5 and NPR3. Fan $Y$ et. al reported that the up-regulator expression of hsa-miR-23a could suppress hdac2 and activate NF-KB and influence the ability of adhesion, invasion and proliferation of trophoblast (36). Our study showed that hsa-miR-23a played an important role in embryo implantation. At the same time, there had several studies suggested that hsa-miR-23a and hsamiR-23b were closely related to MAPK pathway $(37,38)$. As many studies reported, MAPK pathway played an important role in embryo implantation, and it was closely related to the ability of adhesion, invasion and proliferation of trophoblast and the procession of endometrium angiogenesis (39-41). The causal relationship network in Fig. 5 showed that DUSP5 down-regulates MAPK1 $(R=0.42)$. According to these results, we could make a hypothesis that IncRNA PART1 may act as a sponge of hsa-miR-23a/b to down-regulate DUSP5 to promote RIF.

In addition, we found that hsa-miR-96-5p which also highly expressed in serum was also closely related to the ability of adhesion, invasion and proliferation of trophoblast (42). Chen $D$ et. al found that the function of human trophoblast cell could be promoted by down-regulating miR-96-5p via targeting 
DDAH1 (42). Another study confirmed that PTEN, the main negative regulator of PI3K-Akt pathway, was direct target of hsa-miR-96-5p (43). Several studies had shown that abnormal activation of AKT pathway was one of the main mechanisms of endometrial abnormality during WOI period (44-46). Based on the above results, we speculated that hsa-miR-96-5p could affect the receptivity of the endometrium during WOI by activated the PI3K-AKT pathway.

In this study, the results of functional enrichment analysis of miRNAs target genes also supported our conclusions. The targeted mRNAs of hsa-miR-96-5p mainly enriched in the terms of cellular response to organonitrogen compound, negative regulation of cell differentiation, regulation of protein serine/ threonine kinase activity, apoptosis pathway and MAPK signaling pathway.

At the same time, we also developed a non-invasive RIF diagnostic scoring model to assist in the diagnosis and treatment of RIF patients and it showed better predictability and accuracy. As far as we know, this was the first RIF predictive scoring model based on circulating miRNA. Clinical trials of models will also be conducted soon. For this study, there were still some shortcomings, such as lacking adequate laboratory tests to verify the mechanism. Because of the huge work of validation experiments, we are planning to do so, and the results will be published in our future research.

\section{Declarations}

\section{Ethics approval and consent to participate}

Not application.

\section{Consent for publication}

All authors had been carefully examined and agreed to publish.

\section{Availability of data and materials}

The data that support the findings of this study are available in the Gene Expression Omnibus (GEO) database.

\section{Competing interests}

The authors declare that there are no conflicts of interest to disclose.

\section{Funding statement}

Supported by the National Natural Science Foundation of China [grant number 81871214] and National Key R\&D Program of China [grant number 2017YFC1001603].

\section{Author Contributions}


Cong Fang and Peigen Chen carried out the study. Peigen Chen analyzed and interpreted the data. Yingchun Guo and Tingting Li drafted the manuscript. Lei Jia, Yanfang Wang and Yi Zhou collected and analyzed the data. Cong Fang and Peigen Chen coordinated the study, participated in the design and reviewed the manuscript. All authors read and approved the final manuscript.

\section{Acknowledgements}

We thank Dr.Jianming Zeng(University of Macau), and all the members of his bioinformatics team, biotrainee, for generously sharing their experience.

\section{References}

1. Coughlan C, Ledger W, Wang Q, Liu F, Demirol A, Gurgan T, et al. Recurrent implantation failure: definition and management. Reprod Biomed Online. 2014;28(1):14-38.

2. Simon A, Laufer N. Repeated implantation failure: clinical approach. Fertil Steril. 2012;97(5):1039-43.

3. Thornhill AR, deDie-Smulders CE, Geraedts JP, Harper JC, Harton GL, Lavery SA, et al. ESHRE PGD Consortium 'Best practice guidelines for clinical preimplantation genetic diagnosis (PGD) and preimplantation genetic screening (PGS)'. Hum Reprod. 2005;20(1):35-48.

4. Cha J, Sun X, Dey SK. Mechanisms of implantation: strategies for successful pregnancy. Nat Med. 2012;18(12):1754-67.

5. Bagga S, Bracht J, Hunter S, Massirer K, Holtz J, Eachus R, et al. Regulation by let-7 and lin-4 miRNAs results in target mRNA degradation. Cell. 2005;122(4):553-63.

6. Bartel DP. MicroRNAs: genomics, biogenesis, mechanism, and function. Cell. 2004;116(2):281-97.

7. Galliano D, Pellicer A. MicroRNA and implantation. Fertil Steril. 2014;101(6):1531-44.

8. Altmae S, Martinez-Conejero JA, Esteban FJ, Ruiz-Alonso M, Stavreus-Evers A, Horcajadas JA, et al. MicroRNAs miR-30b, miR-30d, and miR-494 regulate human endometrial receptivity. Reprod Sci. 2013;20(3):308-17.

9. Creighton CJ, Benham AL, Zhu H, Khan MF, Reid JG, Nagaraja AK, et al. Discovery of novel microRNAs in female reproductive tract using next generation sequencing. PLoS One. 2010;5(3):e9637.

10. Kuokkanen S, Chen B, Ojalvo L, Benard L, Santoro N, Pollard JW. Genomic profiling of microRNAs and messenger RNAs reveals hormonal regulation in microRNA expression in human endometrium. Biol Reprod. 2010;82(4):791-801.

11. Revel A, Achache H, Stevens J, Smith Y, Reich R. MicroRNAs are associated with human embryo implantation defects. Hum Reprod. 2011;26(10):2830-40.

12. Tan Q, Shi S, Liang J, Zhang X, Cao D, Wang Z. MicroRNAs in Small Extracellular Vesicles Indicate Successful Embryo Implantation during Early Pregnancy. Cells. 2020;9(3).

13. Zhang Q, Ni T, Dang Y, Ding L, Jiang J, Li J, et al. MiR-148a-3p may contribute to flawed decidualization in recurrent implantation failure by modulating HOXC8. J Assist Reprod Genet. 
2020;37(10):2535-44.

14. Rekker K, Saare M, Roost AM, Salumets A, Peters M. Circulating microRNA Profile throughout the menstrual cycle. PLoS One. 2013;8(11):e81166.

15. Chen X, Ba Y, Ma L, Cai X, Yin Y, Wang K, et al. Characterization of microRNAs in serum: a novel class of biomarkers for diagnosis of cancer and other diseases. Cell Res. 2008;18(10):997-1006.

16. Arroyo JD, Chevillet JR, Kroh EM, Ruf IK, Pritchard CC, Gibson DF, et al. Argonaute2 complexes carry a population of circulating microRNAs independent of vesicles in human plasma. Proc Natl Acad Sci U S A. 2011;108(12):5003-8.

17. Pigati L, Yaddanapudi SC, lyengar R, Kim DJ, Hearn SA, Danforth D, et al. Selective release of microRNA species from normal and malignant mammary epithelial cells. PLoS One. 2010;5(10):e13515.

18. Azhari F, Pence S, Hosseini MK, Balci BK, Cevik N, Bastu E, et al. The role of the serum exosomal and endometrial microRNAs in recurrent implantation failure. J Matern Fetal Neonatal Med. 2020:1-11.

19. Salmena L, Poliseno L, Tay Y, Kats L, Pandolfi PP. A ceRNA hypothesis: the Rosetta Stone of a hidden RNA language? Cell. 2011;146(3):353-8.

20. Ritchie ME, Phipson B, Wu D, Hu Y, Law CW, Shi W, et al. limma powers differential expression analyses for RNA-sequencing and microarray studies. Nucleic Acids Res. 2015;43(7):e47.

21. Love MI, Huber W, Anders S. Moderated estimation of fold change and dispersion for RNA-seq data with DESeq2. Genome Biol. 2014;15(12):550.

22. Langfelder P, Horvath S. WGCNA: an R package for weighted correlation network analysis. BMC Bioinformatics. 2008;9:559.

23. Chen $Y$, Wang X. miRDB: an online database for prediction of functional microRNA targets. Nucleic Acids Res. 2020;48(D1):D127-D31.

24. Hsu SD, Lin FM, Wu WY, Liang C, Huang WC, Chan WL, et al. miRTarBase: a database curates experimentally validated microRNA-target interactions. Nucleic Acids Res. 2011;39(Database issue):D163-9.

25. Agarwal V, Bell GW, Nam JW, Bartel DP. Predicting effective microRNA target sites in mammalian mRNAs. Elife. 2015;4.

26. Teng X, Chen X, Xue H, Tang Y, Zhang P, Kang Q, et al. NPInter v4.0: an integrated database of ncRNA interactions. Nucleic Acids Res. 2020;48(D1):D160-D5.

27. Paraskevopoulou MD, Vlachos IS, Karagkouni D, Georgakilas G, Kanellos I, Vergoulis T, et al. DIANALncBase v2: indexing microRNA targets on non-coding transcripts. Nucleic Acids Res. 2016;44(D1):D231-8.

28. Bader GD, Hogue CW. An automated method for finding molecular complexes in large protein interaction networks. BMC Bioinformatics. 2003;4:2.

29. Zhou Y, Zhou B, Pache L, Chang M, Khodabakhshi AH, Tanaseichuk O, et al. Metascape provides a biologist-oriented resource for the analysis of systems-level datasets. Nat Commun. 
2019;10(1):1523.

30. Han H, Shim H, Shin D, Shim JE, Ko Y, Shin J, et al. TRRUST: a reference database of human transcriptional regulatory interactions. Sci Rep. 2015;5:11432.

31. Lo Surdo P, Calderone A, lannuccelli M, Licata L, Peluso D, Castagnoli L, et al. DISNOR: a disease network open resource. Nucleic Acids Res. 2018;46(D1):D527-D34.

32. Therneau TM. A Package for Survival Analysis in S. 2015.

33. Kang YJ, Lees M, Matthews LC, Kimber SJ, Forbes K, Aplin JD. MiR-145 suppresses embryo-epithelial juxtacrine communication at implantation by modulating maternal IGF1R. J Cell Sci. 2015;128(4):804-14.

34. Shah RV, Rong J, Larson MG, Yeri A, Ziegler O, Tanriverdi K, et al. Associations of Circulating Extracellular RNAs With Myocardial Remodeling and Heart Failure. JAMA Cardiol. 2018;3(9):871-6.

35. Nappi L, Thi M, Adra N, Hamilton RJ, Leao R, Lavoie JM, et al. Integrated Expression of Circulating miR375 and miR371 to Identify Teratoma and Active Germ Cell Malignancy Components in Malignant Germ Cell Tumors. Eur Urol. 2021;79(1):16-9.

36. Fan Y, Dong Z, Zhou G, Fu J, Zhan L, Gao M, et al. Elevated miR-23a impairs trophoblast migration and invasiveness through HDAC2 inhibition and NF-kappaB activation. Life Sci. 2020;261:118358.

37. Guo Y, Min Z, Jiang C, Wang W, Yan J, Xu P, et al. Downregulation of HS6ST2 by miR-23b-3p enhances matrix degradation through p38 MAPK pathway in osteoarthritis. Cell Death Dis. 2018;9(6):699.

38. Ma M, Dai J, Tang H, Xu T, Yu S, Si L, et al. MicroRNA-23a-3p Inhibits Mucosal Melanoma Growth and Progression through Targeting Adenylate Cyclase 1 and Attenuating cAMP and MAPK Pathways. Theranostics. 2019;9(4):945-60.

39. Goryszewska E, Kaczynski P, Balboni G, Waclawik A. Prokineticin 1-prokineticin receptor 1 signaling promotes angiogenesis in the porcine endometrium during pregnancydagger. Biol Reprod. 2020;103(3):654-68.

40. Zhang L, Liu X, Che S, Cui J, Ma X, An X, et al. Endometrial Epithelial Cell Apoptosis Is Inhibited by a ciR8073-miR181a-Neurotensis Pathway during Embryo Implantation. Mol Ther Nucleic Acids. 2019;14:262-73.

41. Baryla M, Kaczynski P, Goryszewska E, Riley SC, Waclawik A. Prostaglandin F2alpha stimulates adhesion, migration, invasion and proliferation of the human trophoblast cell line HTR-8/SVneo. Placenta. 2019;77:19-29.

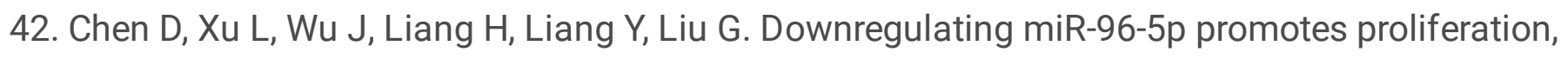
migration, and invasion, and inhibits apoptosis in human trophoblast cells via targeting DDAH1. Reprod Biol. 2020;21(1):100474.

43. Vahabi M, Pulito C, Sacconi A, Donzelli S, D'Andrea M, Manciocco V, et al. miR-96-5p targets PTEN expression affecting radio-chemosensitivity of HNSCC cells. J Exp Clin Cancer Res. 2019;38(1):141. 
44. Makker A, Goel MM, Nigam D, Mahdi AA, Das V, Agarwal A, et al. Aberrant Akt Activation During Implantation Window in Infertile Women With Intramural Uterine Fibroids. Reprod Sci. 2018;25(8):1243-53.

45. Zhang L, Liu X, Liu J, Ma X, Zhou Z, Song Y, et al. miR-26a promoted endometrial epithelium cells (EECs) proliferation and induced stromal cells (ESCs) apoptosis via the PTEN-PI3K/AKT pathway in dairy goats. J Cell Physiol. 2018;233(6):4688-706.

46. Liu L, Wang Y, Yu Q. The PI3K/Akt signaling pathway exerts effects on the implantation of mouse embryos by regulating the expression of RhoA. Int J Mol Med. 2014;33(5):1089-96.

\section{Tables}

Due to technical limitations, table 1 is only available as a download in the Supplemental Files section.

\section{Figures}

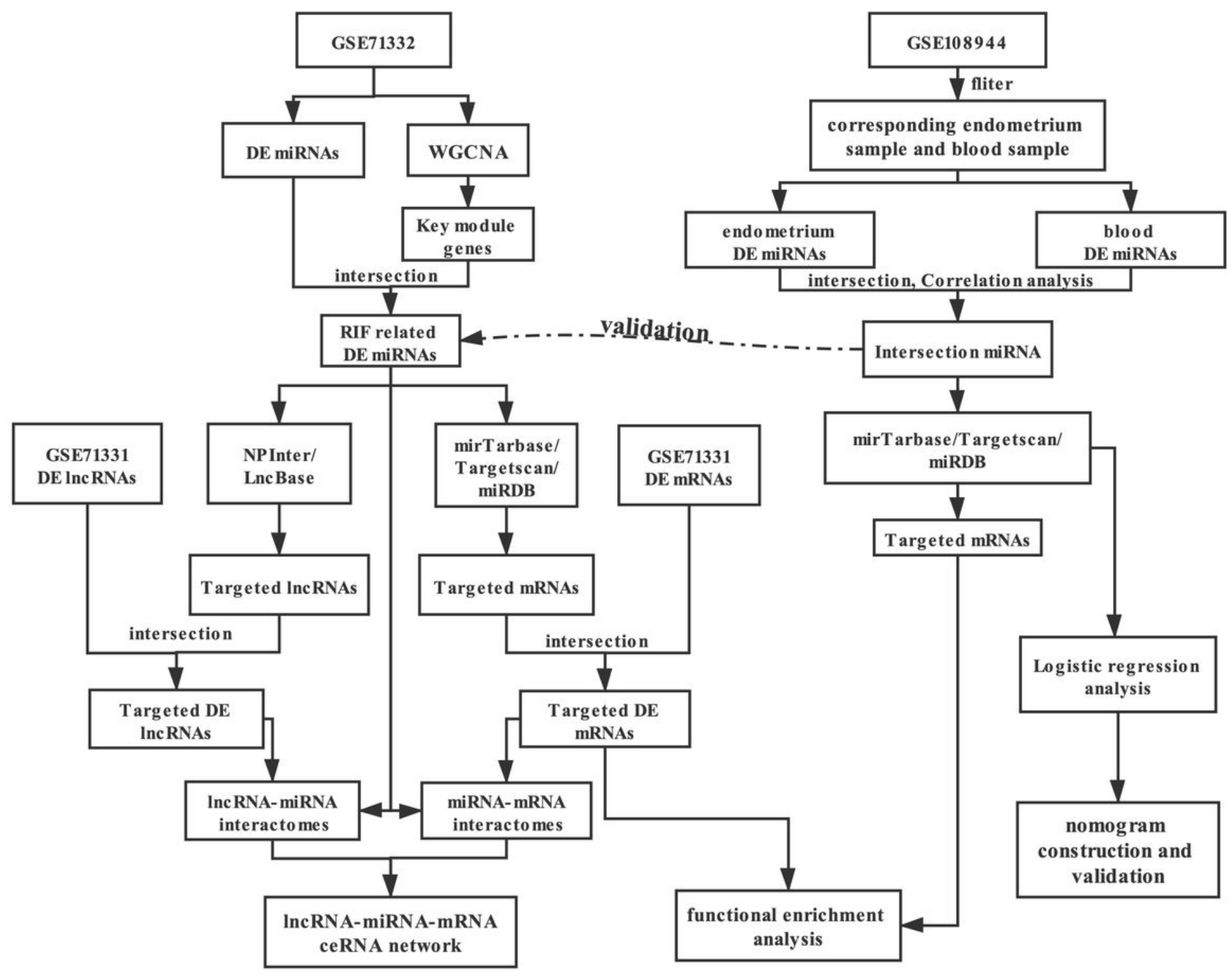


Figure 1

The workflow of this study.

A

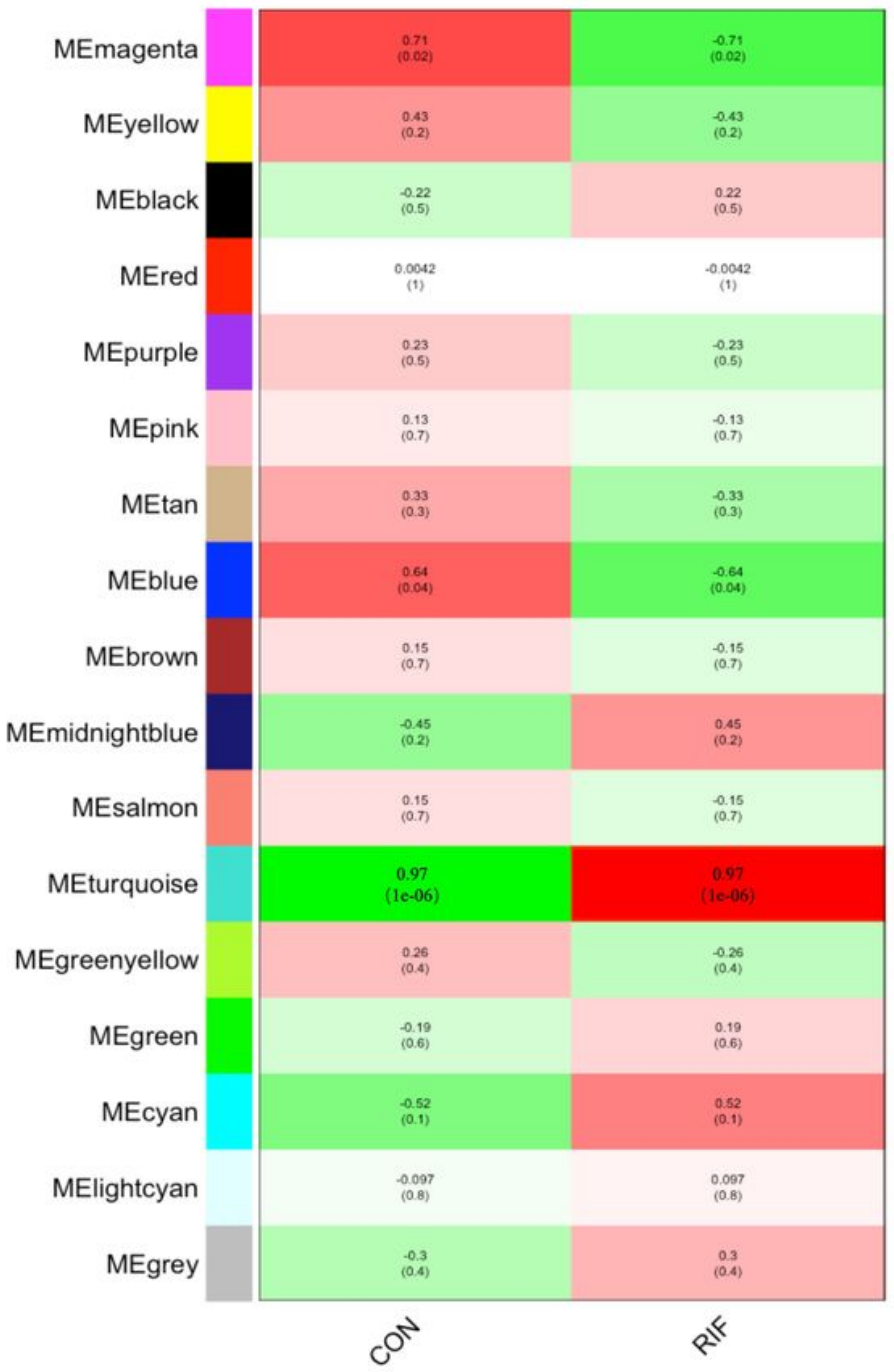

B

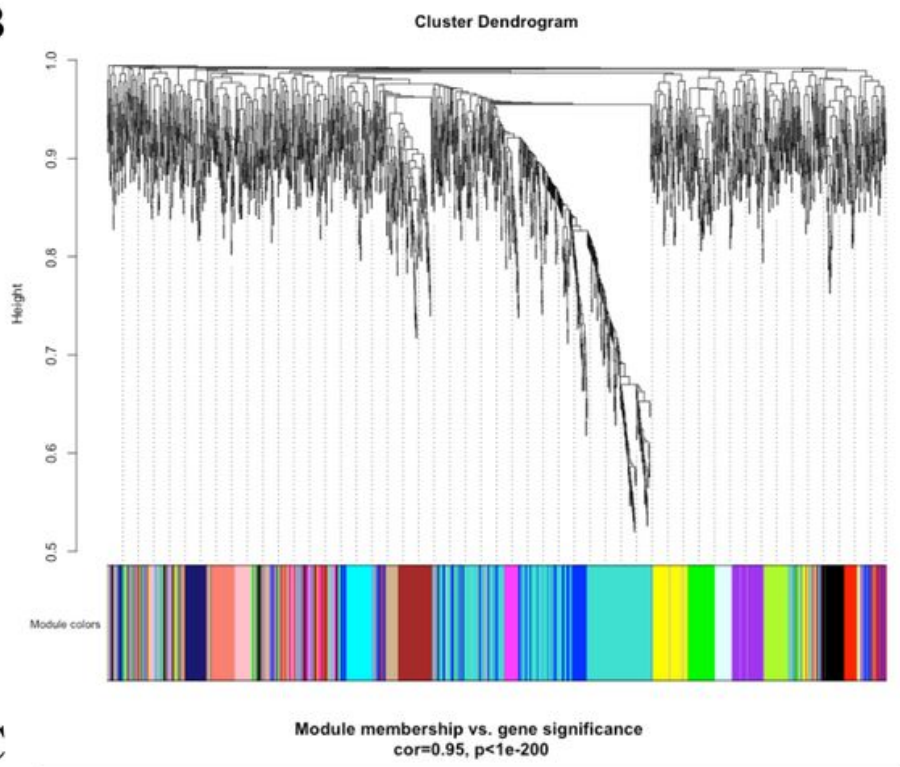

${ }^{-0} \mathrm{C}$

$\stackrel{-}{\circ}$

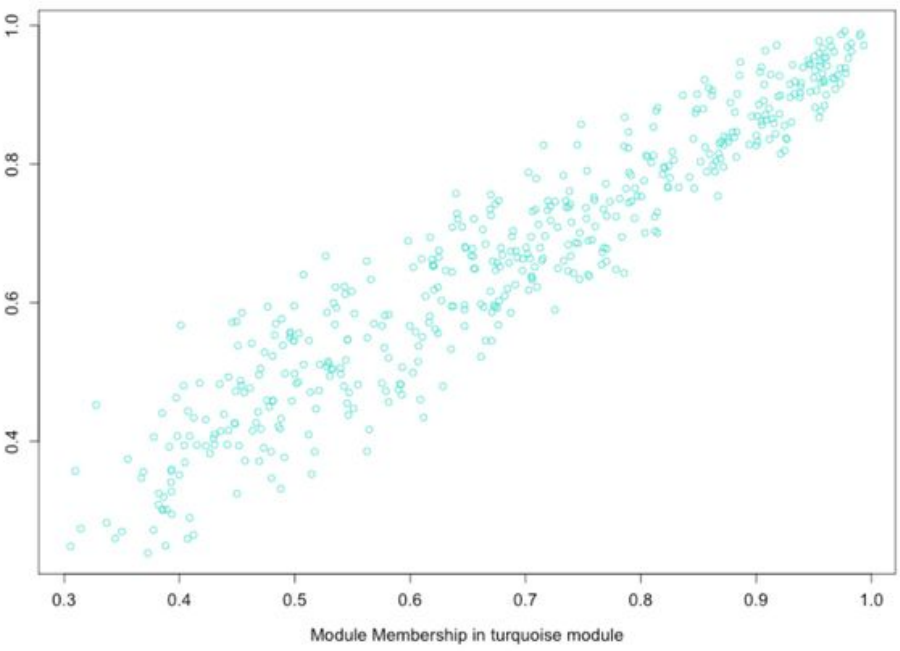

\section{Figure 2}

Weighted gene correlation network analysis. A. The relationships between the corresponding modules and clinical phenotypes; B. The modules selected with a minimum module size of 50 for further analysis; C. The module membership in turquoise module. 

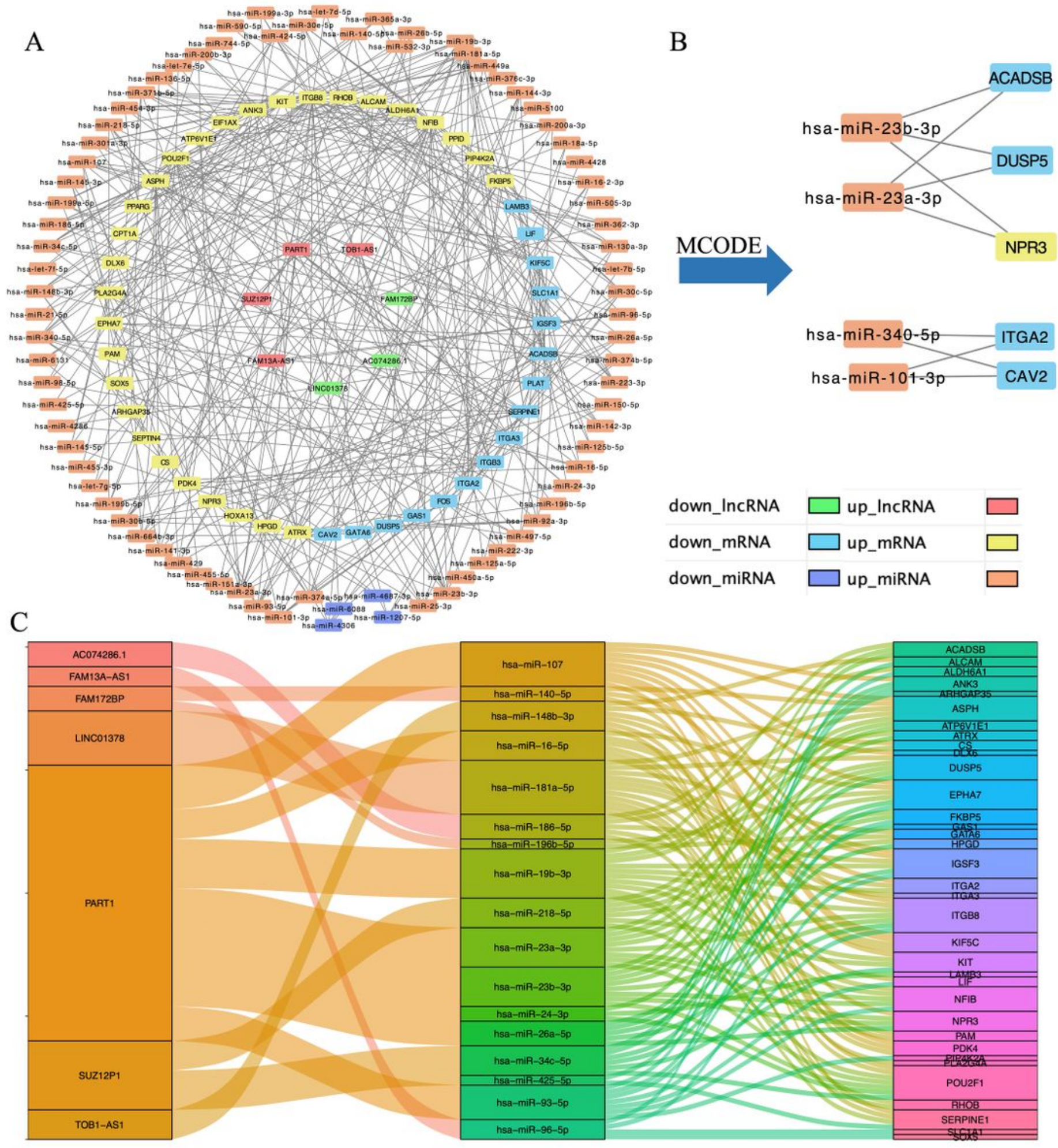

Figure 3

Construction of IncRNA-miRNA-mRNA regulatory network. A. The IncRNA-miRNA-mRNA regulatory network; B. The key module network selected from IncRNA-miRNA-mRNA regulatory network; C. The connection between IncRNAs, miRNAs and mRNAs. 

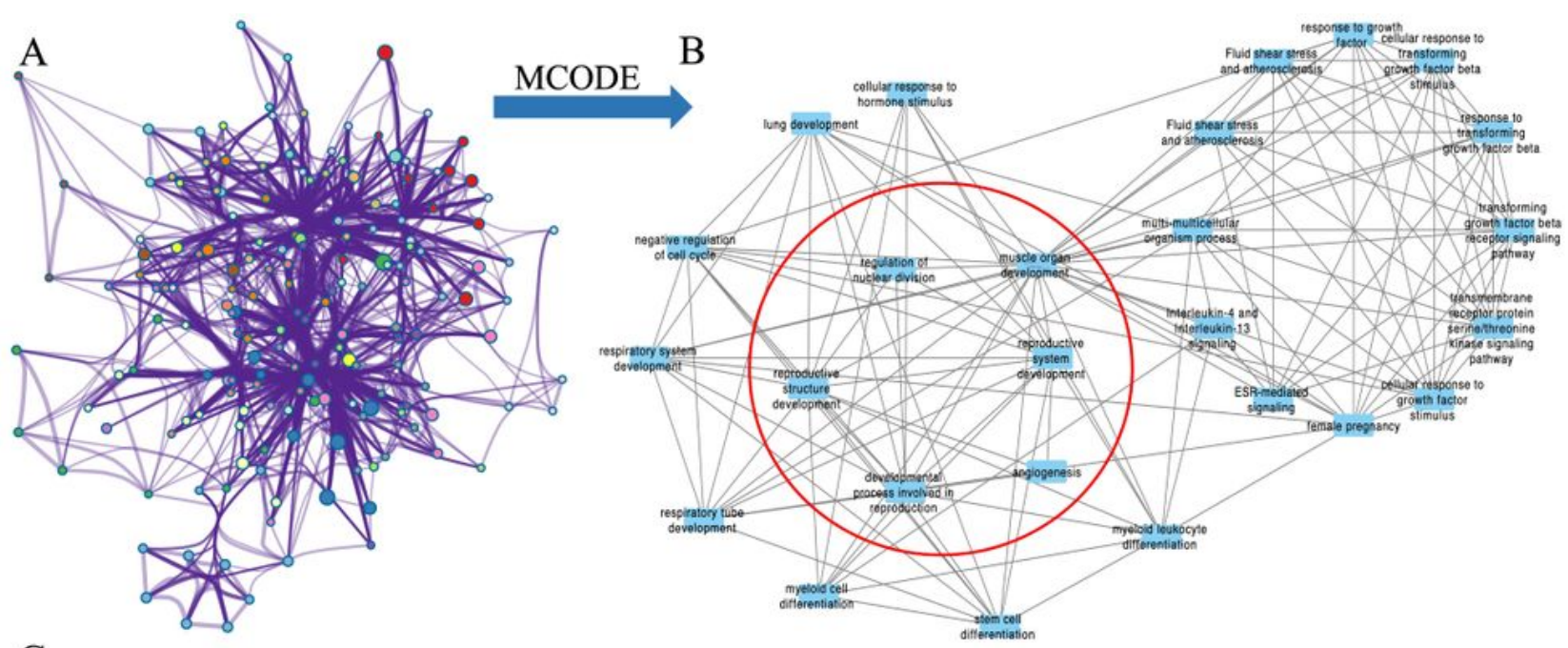

C

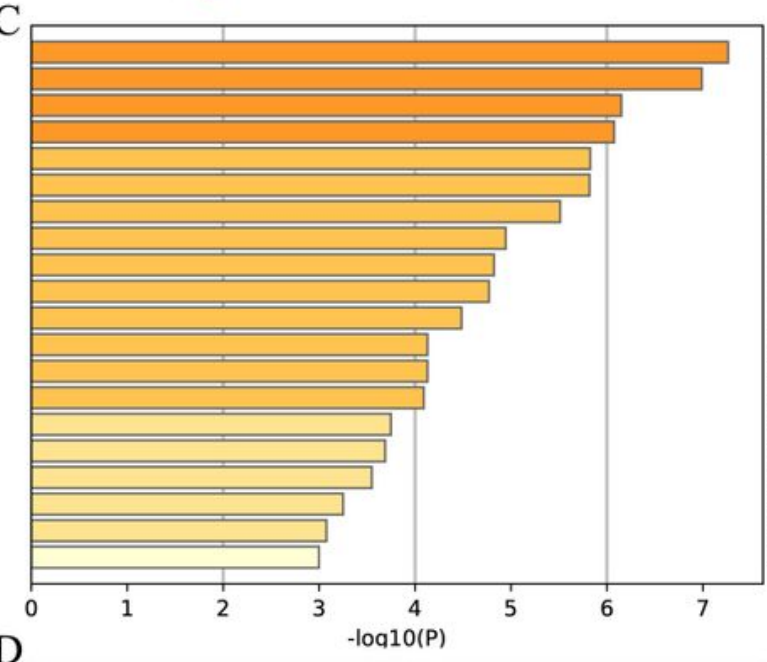

GO:0001704: formation of primary germ layer

GO:0033627: cell adhesion mediated by integrin

GO:0007565: female pregnancy

GO:0006935: chemotaxis

GO:0050878: regulation of body fluid levels

GO:0001501: skeletal system development

GO:0006631: fatty acid metabolic process

GO:0032870: cellular response to hormone stimulus

GO:0048514: blood vessel morphogenesis

GO:0071363: cellular response to growth factor stimulus

GO:0033218: amide binding

GO:0048608: reproductive structure development

GO:0048732: gland development

GO:1902893: regulation of pri-miRNA transcription by RNA polymerase II

GO:0010942: positive regulation of cell death

GO:0048736: appendage development

GO:0010035: response to inorganic substance

GO:0061640: cytoskeleton-dependent cytokinesis

GO:0050730: regulation of peptidyl-tyrosine phosphorylation

GO:0045165: cell fate commitment

D

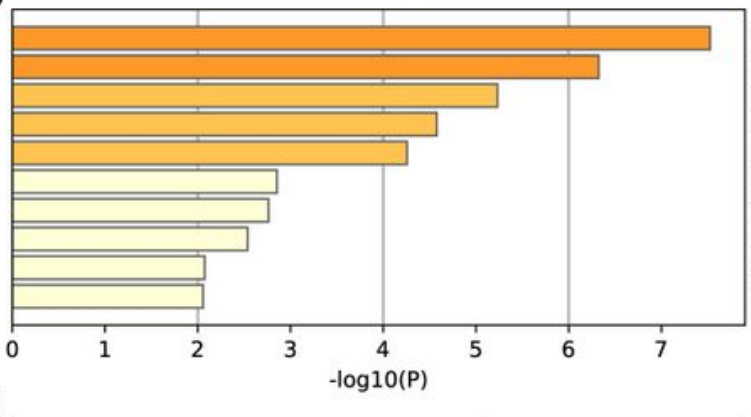

ko04510: Focal adhesion

R-HSA-9006931: Signaling by Nuclear Receptors

R-HSA-109582: Hemostasis

R-HSA-422475: Axon guidance

M186: PID PDGFRB PATHWAY

M145: PID P53 DOWNSTREAM PATHWAY

hsa04010: MAPK signaling pathway

R-HSA-8978868: Fatty acid metabolism

ko04144: Endocytosis

R-HSA-1257604: PIP3 activates AKT signaling

E

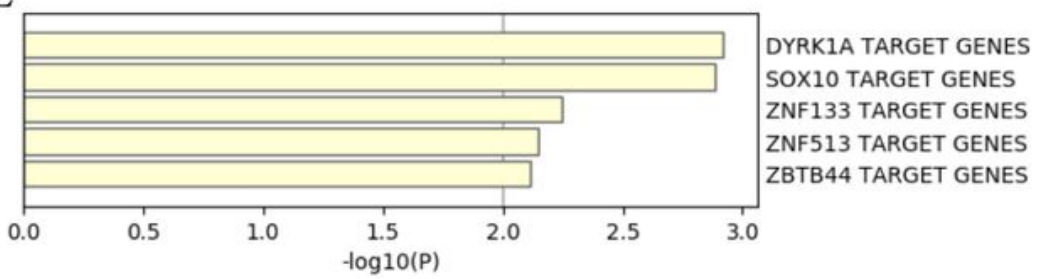

Figure 4

Functional enrichment analysis of targeted DE mRNAs of GSE71331/71332. A. The network of functional terms; B. The key module network selected by MCODE (inside the red circle) and first neighbor node of the key module network (outside the red circle); C. The enriched GO terms of targeted DE mRNAs; D. The enriched pathway of targeted DE mRNAs; E. The transcription factors related to targeted DE mRNAs. 


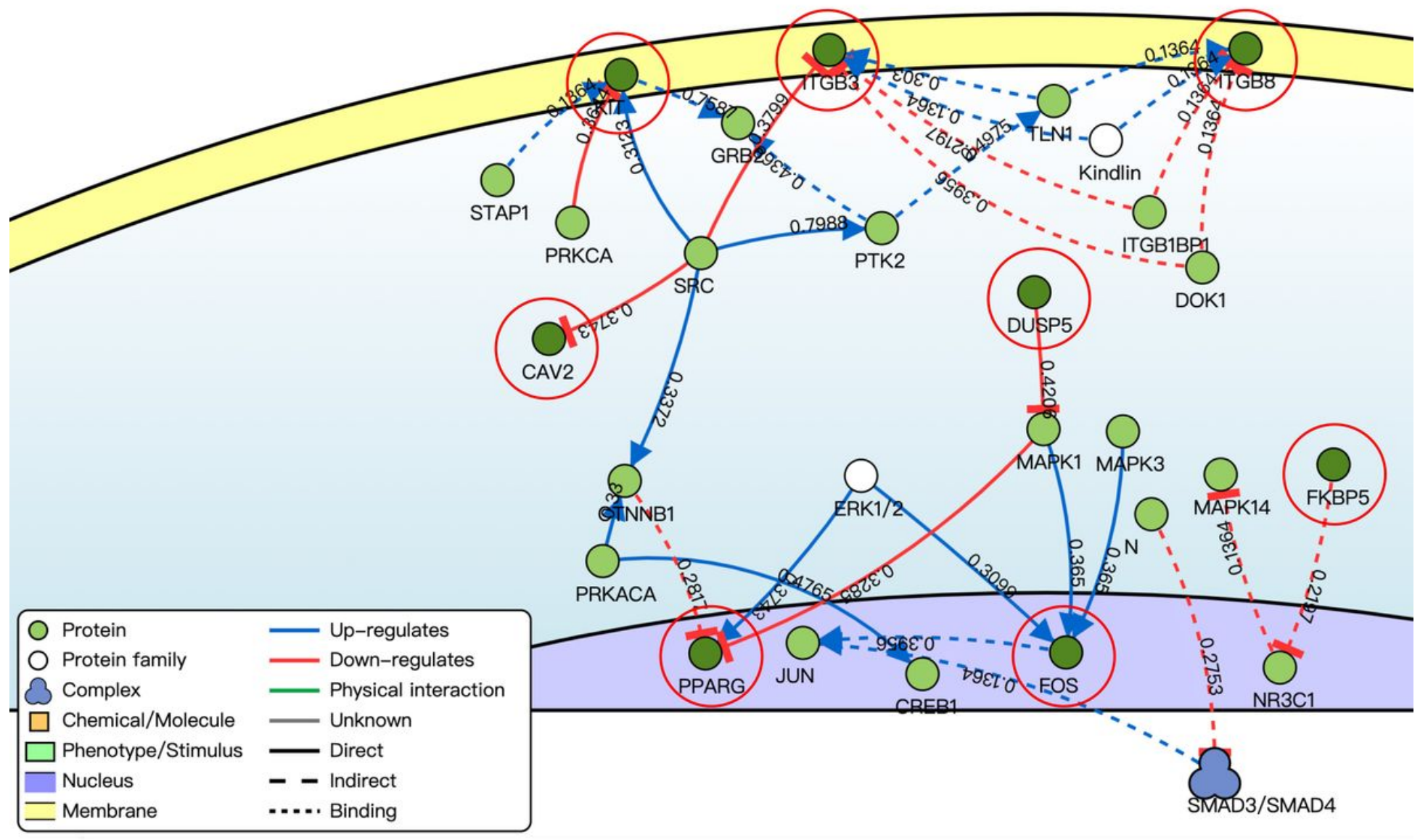

Figure 5

Causal interaction analysis of targeted DE mRNAs of GSE71331/71332 (inside the red circle) and their first neighbor genes (outside the red circle) by using DisNor. The numbers on the line stand for correlation coefficient. 

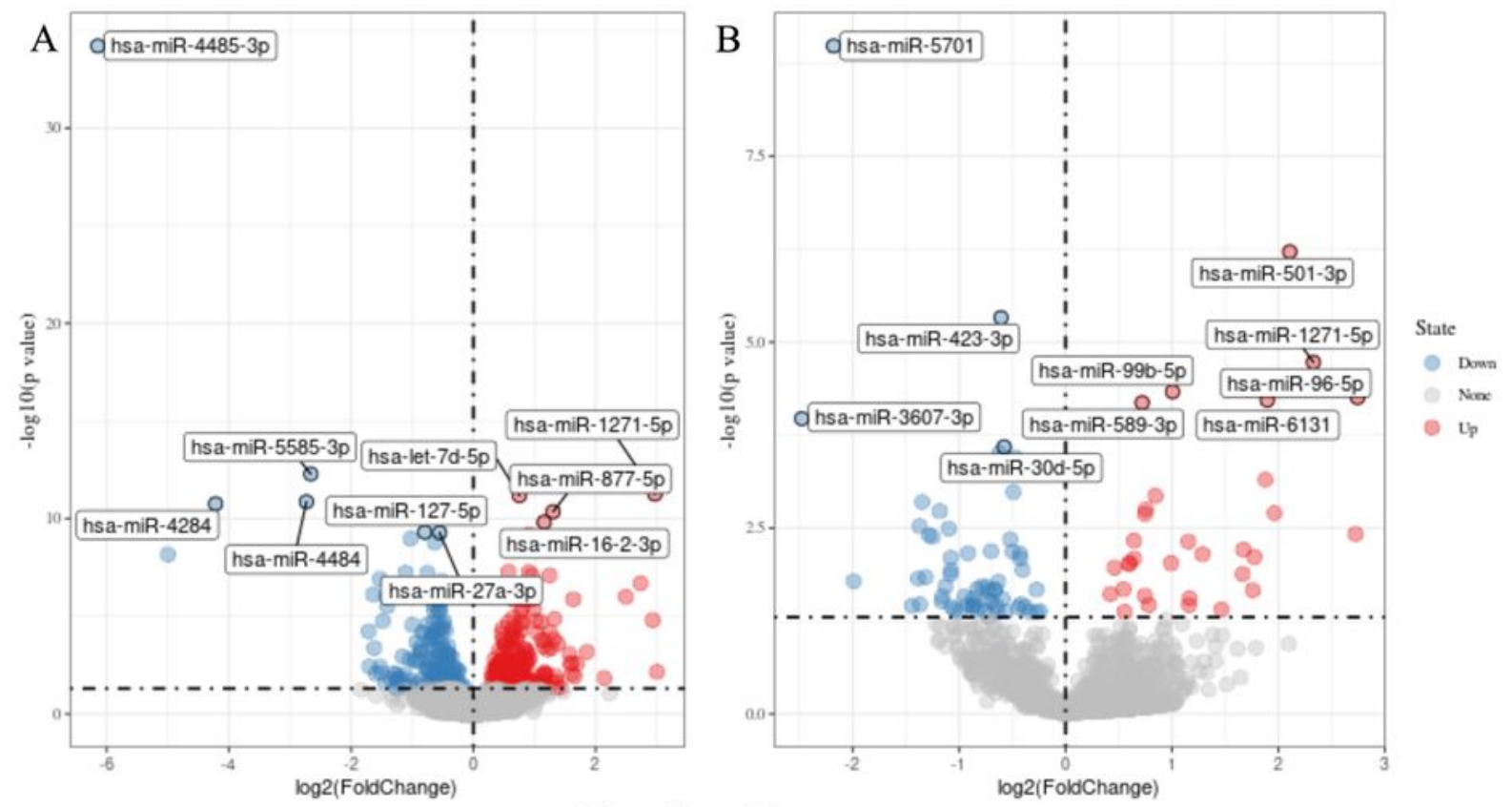

$\mathrm{C}$
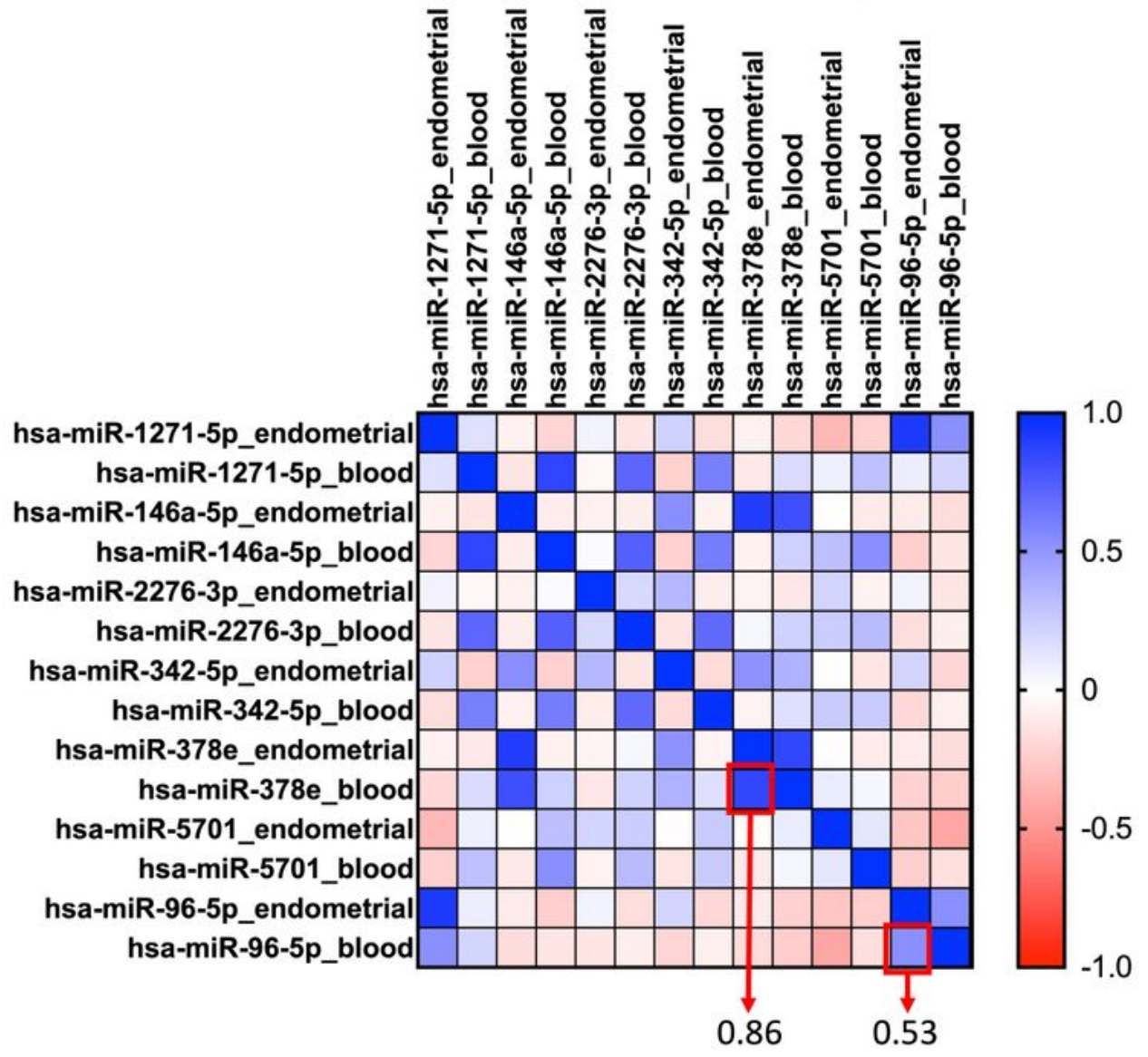

Figure 6

Selection of co-expression miRNAs between serum and endometrial samples. A. The volcano plot of differentially expressed genes in endometrium sample of GSE108966; B. The volcano plot of differentially expressed genes in serum sample of GSE108966; C. The heatmap of the co-expression miRNAs between serum and endometrial samples. The numbers inside the boxes stand for correlation coefficient. 


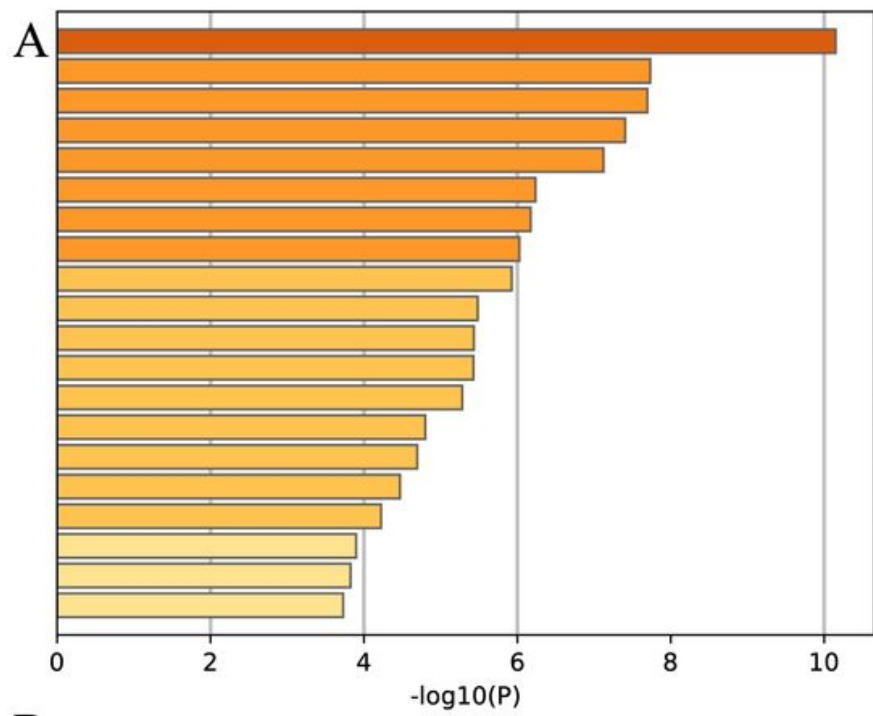

GO:0071417: cellular response to organonitrogen compound GO:0045596: negative regulation of cell differentiation GO:0071900: regulation of protein serine/threonine kinase activity GO:0009896: positive regulation of catabolic process GO:0035690: cellular response to drug GO:0070997: neuron death

GO:0044772: mitotic cell cycle phase transition GO:0007420: brain development

GO:0080135: regulation of cellular response to stress GO:0060562: epithelial tube morphogenesis

GO:0045444: fat cell differentiation

GO:0071363: cellular response to growth factor stimulus

GO:0048598: embryonic morphogenesis

GO:0007565: female pregnancy

GO:0043161: proteasome-mediated ubiquitin-dependent protein catabolic process GO:0003682: chromatin binding

GO:0061351: neural precursor cell proliferation

GO:0030036: actin cytoskeleton organization

GO:0007268: chemical synaptic transmission

GO:0048511: rhythmic process

B

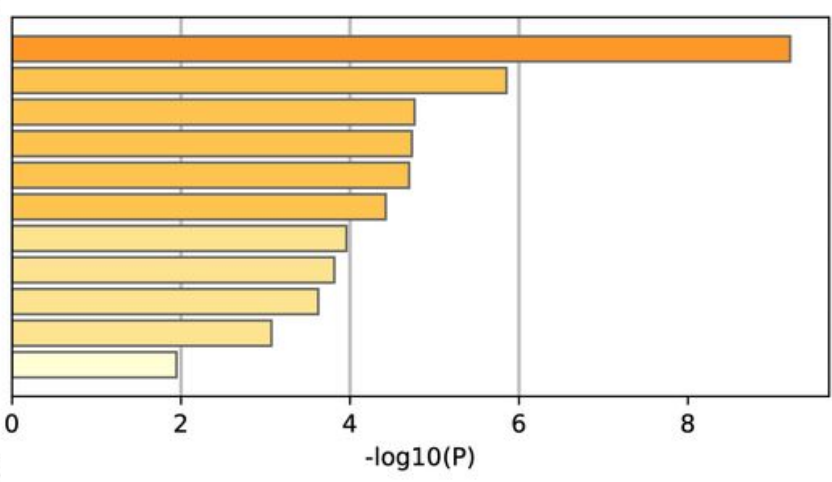

hsa04211: Longevity regulating pathway

hsa05202: Transcriptional misregulation in cancer

ko05206: MicroRNAs in cancer

ko04722: Neurotrophin signaling pathway

R-HSA-9006925: Intracellular signaling by second messengers

ko04210: Apoptosis

hsa04022: cGMP-PKG signaling pathway

hsa05166: Human T-cell leukemia virus 1 infection

hsa04010: MAPK signaling pathway

R-HSA-112315: Transmission across Chemical Synapses

R-HSA-1640170: Cell Cycle

$\mathrm{C}$

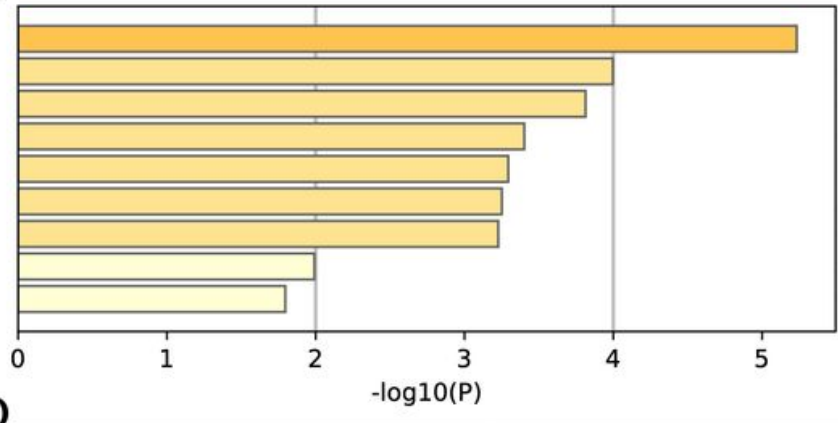

GO:0004712: protein serine/threonine/tyrosine kinase activity GO:0046620: regulation of organ growth GO:0006913: nucleocytoplasmic transport GO:0050804: modulation of chemical synaptic transmission ko05203: Viral carcinogenesis

GO:0071383: cellular response to steroid hormone stimulus GO:0006888: ER to Golgi vesicle-mediated transport GO:1902532: negative regulation of intracellular signal transduction GO:0043065: positive regulation of apoptotic process

$\mathrm{D}$

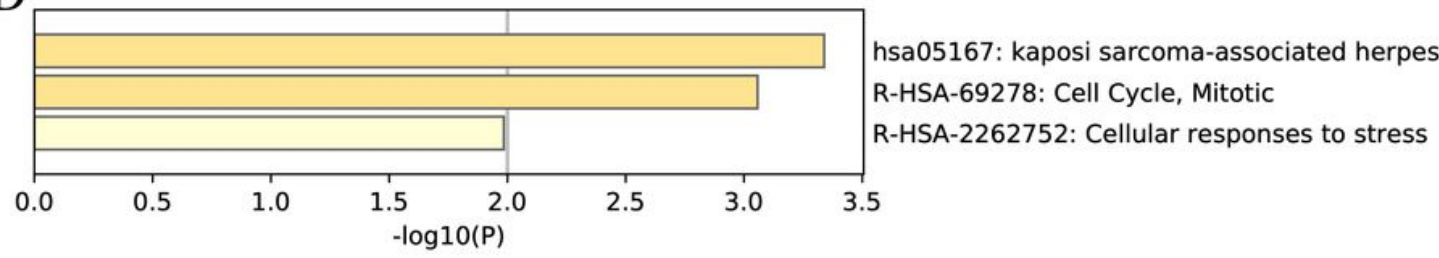

Figure 7

Functional enrichment analysis of the target mRNAs of hsa-miR-378e and hsa-miR-96-5p. The enriched GO terms (A) and pathways (B) of the target mRNAs of hsa-miR-96-5p. The enriched GO terms (C) and pathways (D) of the target mRNAs of hsa- hsa-miR-378e. 
A

Points

20

30

40

50

60

70

80

90

100

age

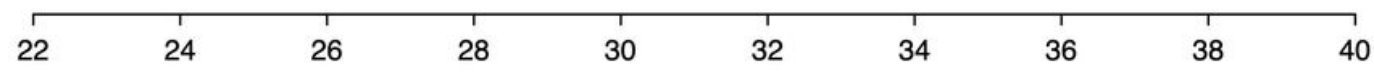

hsa.miR.378e_blood

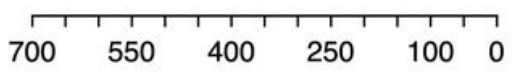

hsa.miR.96.5p_blood

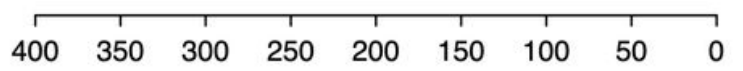

Total Points

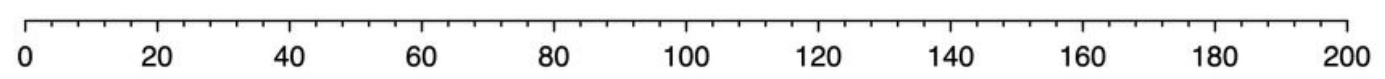

Risk

B

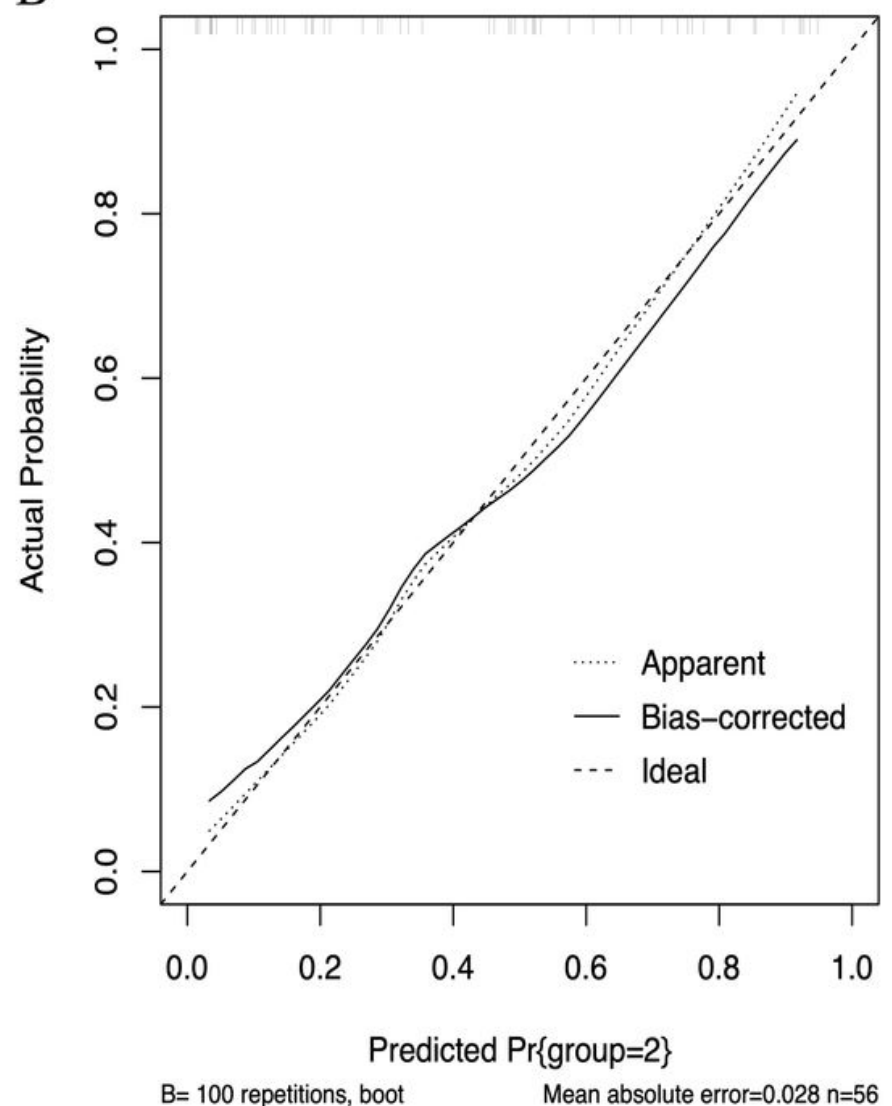

C

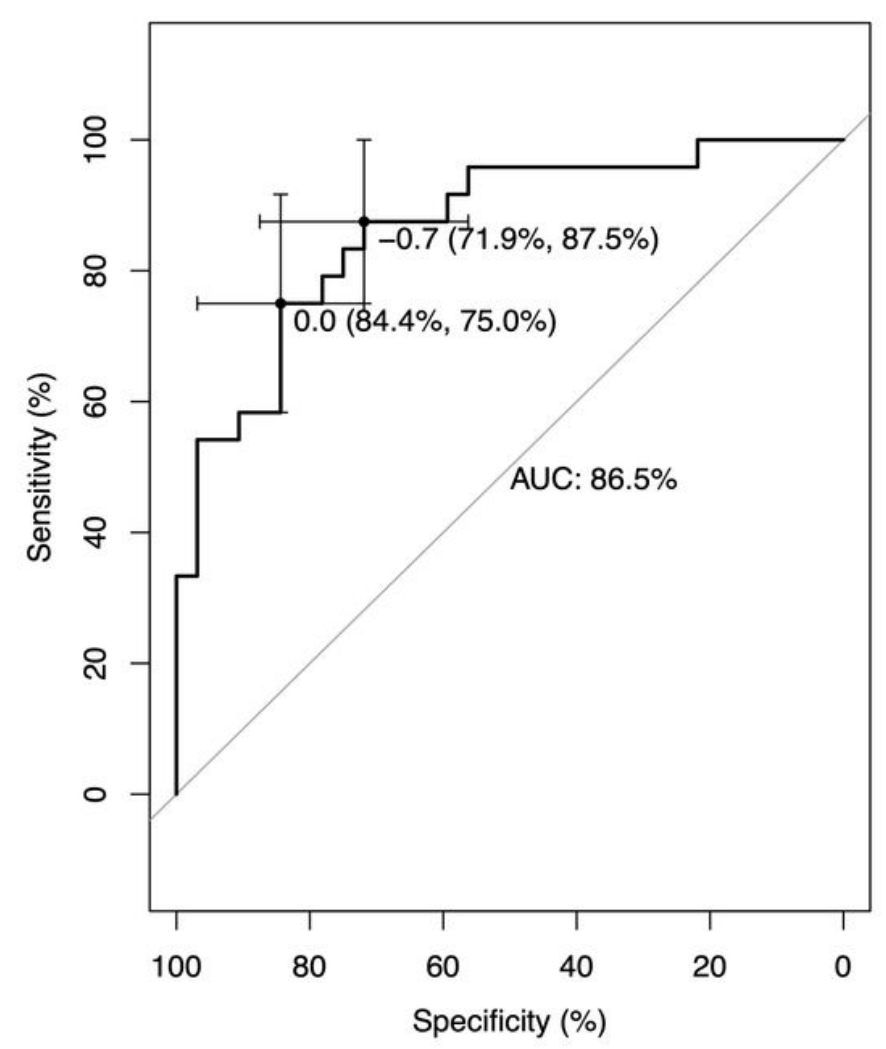

\section{Figure 8}

The prediction model based on circulating miRNAs. A. The nomogram of the prediction models based on circulating miRNAs; $\mathrm{B}$. The calibration curves of the prediction model; $\mathrm{C}$. The receiver operating characteristic (ROC) curve of the prediction model.

\section{Supplementary Files}


This is a list of supplementary files associated with this preprint. Click to download.

- Table1clinicalcharacteristicofGSE71331.xIsx

- spplement.docx

- supplementtable1.xlsx

- supplementtable2.xlsx

- supplementtable3.xlsx

- supplementtable4.xlsx

- supplementtable5.xlsx 